\title{
Poliquetos pelágicos (Annelida, Polychaeta) del Pacífico suroriental frente a Chile e islas oceánicas
}

\author{
Nicolás Rozbaczylo ${ }^{1}$, Rodrigo A. Moreno ${ }^{2}$, Guillermo Guzmán ${ }^{2}$ \& Jeannelle Jaque ${ }^{2}$ \\ ${ }^{1}$ Departamento de Ecología, Facultad de Ciencias Biológicas, \\ Pontificia Universidad Católica de Chile. Casilla 114-D, Santiago, Chile \\ ${ }^{2}$ Museo del Mar, Departamento de Ciencias del Mar, \\ Universidad Arturo Prat. Casilla 121, Iquique, Chile
}

\begin{abstract}
RESUMEN. Durante los cruceros de investigación Cimar 5 y Cimar 6, en aguas del Pacífico suroriental frente a Chile e islas oceánicas (isla de Pascua, San Félix, San Ambrosio y archipiélago de Juan Fernández), se recolectaron mediante una red de arrastre horizontal y vertical IKMT, un total de 109 poliquetos pelágicos pertenecientes a 12 especies de cuatro familias. En el total de las muestras analizadas se determinaron las especies Torrea pelagica, Vanadis crystallina, V. formosa, V. longissima, V. minuta, Watelio gravieri, Lopadorhynchus uncinatus, Pelagobia longicirrata, Enapteris euchaeta, Tomopteris carpenterii, T. septentrionalis y Drieschia nans. Todos los taxa presentan un patrón de distribución cosmopolita a excepción de Torrea pelagica y Drieschia nans que serían endémicas para el Pacífico sur. Se determinaron seis especies de Alciopidae (50\% de abundancia relativa), tres especies de Tomopteridae (25\%), dos especies de Lopadorhynchidae $(16,7 \%)$ y una especie de Polynoidae $(8,3 \%)$. Se registra por primera vez la presencia en aguas del Pacífico suroriental frente a Chile e islas oceánicas de L. uncinatus, P. longicirrata, V. minuta, V. crystallina y W. gravieri. Con esto el número total de especies de poliquetos pelágicos registradas frente a Chile e islas oceánicas llega a 19. Se discute la necesidad de aumentar los muestreos planctónicos cuantitativos para incrementar el conocimiento acerca de la biología y ecología de los poliquetos pelágicos del Pacífico suroriental, a fin de establecer sus relaciones en la dinámica oceánica del plancton.
\end{abstract}

Palabras clave: Polychaeta, holoplancton, islas oceánicas, isla de Pascua, Chile.

\section{Pelagic polychaetes (Annelida, Polychaeta) from the SE Pacific Ocean off Chile and its' Oceanic Islands}

\begin{abstract}
A total of 109 specimens of pelagic polychaetes belonging to 12 species in four families was collected using an Isaacs Kids Midwater Trawl (IKMT) during the Cimar 5 and Cimar 6 cruises in the SE Pacific Ocean off the Chilean coast as well as Easter I., San Felix I., San Ambrosio I. and the Juan Fernandez Archipelago. The species Torrea pelagica, Vanadis crystallina, V. formosa, V. longissima, V. minuta, Watelio gravieri, Lopadorhynchus uncinatus, Pelagobia longicirrata, Enapteris euchaeta, Tomopteris carpenterii, T. septentrionalis and Drieschia nans were identified from the complete set of plankton samples analysed. All the taxa have a cosmopolitan distribution with the exception of two species, Torrea pelagica and Drieschia nans, which are endemic to the South Pacific Ocean. Six species of Alciopidae (50\% of abundance), three species of Tomopteridae (25\%), two species of Lopadorhynchidae (16.7\%) and one species of Polynoidae (8.3\%) were identified in the samples analyzed. L. uncinatus, P. longicirrata, V. minuta, V. crystallina and W. gravieri are here reported for the first time in the SE Pacific off Chile and its' oceanic islands. Present results suggest the total number of species of pelagic polychaetes off the Chilean coast and its' oceanic islands is at least 19. We discuss the need for increasing quantitative plankton sampling to improve knowledge of the biology and ecology of the pelagic polychaetes of the SE Pacific Ocean, as well as to establish their relationships with the oceanic dynamics of the plankton.
\end{abstract}

Key words: Polychaeta, pelagic species, oceanic islands, Easter Island, Chile.

Autor corresponsal: Nicolás Rozbaczylo (nrozbac@bio.puc.cl) 


\section{INTRODUCCIÓN}

A nivel mundial, los poliquetos pelágicos han sido tradicionalmente agrupados en seis familias: Tomopteridae, Alciopidae, Lopadorhynchidae, Typhloscolecidae, Iospilidae y Pontodoridae (Fauchald, 1977; Pettibone, 1982). Sin embargo, Rouse \& Fauchald (1997), Hutchings (2000) y Rouse \& Pleijel (2001) reconocen un total de siete familias, al incluir a Poeobiidae. Orensanz \& Ramírez (1973), reconocen ocho familias, al considerar también a Polynoidae.

Las relaciones filogenéticas entre estas familias no son claras, fundamentalmente porque los poliquetos pelágicos son considerados como organismos aberrantes, lo que ha llevado a excluirlos de los análisis cladísticos, por provocar resultados espurios en las relaciones filogenéticas con otros poliquetos (Rouse \& Fauchald, 1997).

En el Pacífico suroriental frente a Chile e isla de Pascua, se había registrado un total de 14 especies de poliquetos pelágicos agrupados en tres familias: Tomopteridae, Alciopidae y Polynoidae (Rozbaczylo, 1985; Rozbaczylo et al., 1987). Su conocimiento proviene principalmente de las recolecciones realizadas entre el siglo XIX y primera mitad del siglo XX por las expediciones del RN Vettor Pisani (1882-1884), RN Liguria (1903-1905), U.S. Fish Commission Steamer Albatross (19041905), Carnegie (1928-1929) y Lund University Chile Expedition (1948-1949). El estudio más reciente corresponde al de Rozbaczylo et al. (1987) sobre Tomopteridae recolectados entre Antofagasta y Valparaíso, a bordo del RV Alexander Agassiz (1974).

Los programas de investigación Cimar organizados por el Comité Oceanográfico Nacional, han permitido a través de los cruceros Cimar 5 y 6 Islas Oceánicas, incrementar el conocimiento científico sobre la fauna de estas áreas lejanas y de difícil acceso. El objetivo del presente trabajo es dar a conocer la fauna de poliquetos pelágicos recolectados en las muestras de plancton de ambos cruceros de investigación y entregar antecedentes taxonómicos de cada especie.

\section{MATERIALES Y MÉTODOS}

Los poliquetos se obtuvieron como parte de las muestras de plancton recolectadas para el Proyecto Fauna Mesopelágica durante el desarrollo de los cruceros Cimar 5 y 6 Islas Oceánicas, en un transecto desde Caldera ( $27^{\circ} 20^{\prime} \mathrm{S}$; $70^{\circ} 52^{\prime} \mathrm{W}$ ) hasta isla de Pascua $\left(27^{\circ} 20^{\prime} \mathrm{S} ; 109^{\circ} 40^{\prime} \mathrm{W}\right)$ efectuado en octubre y noviembre de 1999, entre Valparaíso (3300'S; $71^{\circ} 38^{\prime} S$ ), las islas Desventuradas (San Félix y San Ambrosio) (26 $6^{\circ} 17^{\prime} \mathrm{S}-26^{\circ} 25^{\prime} \mathrm{S}$; $79^{\circ} 53^{\prime} \mathrm{S}-80^{\circ} 00^{\prime} \mathrm{S}$ ),

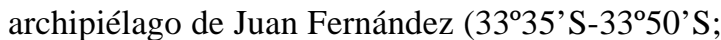
$78^{\circ} 48^{\prime}$ 'S $-80^{\circ} 51^{\prime} S$ ) y Caldera, en septiembre y octubre de 2000, a bordo del buque oceanográfico de la Armada de Chile AGOR Vidal Gormaz (Tabla 1).

Las muestras se obtuvieron mediante una red de arrastre horizontal y vertical Isaacs Kids Midwater Trawl (IKMT) modificada de $9 \mathrm{~m}^{2}$ de abertura de boca, túnel de $10 \mathrm{~m}$ de largo con malla de $10 \mathrm{~mm}$ y copo de abertura de red de $1000 \mathrm{~mm}$ y posteriormente preservadas en una solución de formalina en agua de mar filtrada al 10\% neutralizada con bórax.

Los poliquetos contenidos en cada una de las muestras fueron extraídos bajo lupa binocular y transferidos a una solución de etanol al 70\% para su preservación. En la determinación taxonómica se utilizó principalmente los trabajos de Chamberlin (1919), Dales (1957), Tebble (1960, 1962), Day (1967) y Rozbaczylo et al. (1987). Los ejemplares de Tomopteridae fueron teñidos con una solución de hematoxilina para examinar sus características específicas bajo lupa binocular y los parápodos fueron montados en portaobjetos para su observación microscópica.

Los ejemplares de cada especie se midieron con un ocular graduado, considerando la longitud total desde el extremo anterior del prostomio hasta el final del cuerpo y el ancho máximo desde el extremo distal de las setas opuestas de cada parápodo. El material estudiado se encuentra depositado en el Museo del Mar del Departamento de Ciencias del Mar de la Universidad Arturo Prat de Iquique, Chile (MUAP).

\section{RESULTADOS}

En ambos cruceros se recolectó un total de 109 ejemplares de poliquetos pertenecientes a 12 especies agrupadas en cuatro familias. En las muestras analizadas se determinó un total de seis especies de Alciopidae (50\% de abundancia en las muestras), tres especies de Tomopteridae (25\%), dos especies de Lopadorhynchidae $(16,7 \%)$ y una especie de Polynoidae $(8,3 \%)$. En el crucero Cimar 5 se recolectó un total de 90 poliquetos, destacándose el Tomopteridae Tomopteris septentrionalis 
Tabla 1. Datos de las estaciones del crucero Cimar 5 y Cimar 6 con ocurrencia de poliquetos pelágicos.

Table 1. Data on stations of Cimar 5 and Cimar 6 cruises with ocurrence of pelagic polychaetes.

\begin{tabular}{|c|c|c|c|c|}
\hline Estación & Latitud (S) & Longitud (W) & Fecha & Profundidad (m) \\
\hline \multicolumn{5}{|l|}{ Cimar 5} \\
\hline E-52 & $6^{\circ} 59^{\prime}$ & $71^{\circ} 46^{\prime}$ & 16/10/1999 & 700 \\
\hline E-8 & $27^{\circ} 00^{\prime}$ & $74^{\circ} 35^{\prime}$ & 17/10/1999 & 700 \\
\hline E-9 & $27^{\circ} 03^{\prime}$ & $76^{\circ} 05^{\prime}$ & 18/10/1999 & 700 \\
\hline E-16 & $27^{\circ} 00^{\prime}$ & $86^{\circ} 33^{\prime}$ & 21/10/1999 & 700 \\
\hline E-21 & $27^{\circ} 00^{\prime}$ & $94^{\circ} 05^{\prime}$ & 24/10/1999 & 700 \\
\hline E-23 & $26^{\circ} 59^{\prime}$ & $97^{\circ} 05^{\prime}$ & 25/10/1999 & 700 \\
\hline E-27 & $27^{\circ} 00^{\prime}$ & $103^{\circ} 05^{\prime}$ & 26/10/1999 & 700 \\
\hline E-30 & $26^{\circ} 59^{\prime}$ & $107^{\circ} 35^{\prime}$ & 28/10/1999 & 700 \\
\hline E-35 & $27^{\circ} 11^{\prime}$ & $109^{\circ} 17^{\prime}$ & 04/10/1999 & 700 \\
\hline E-43 & $27^{\circ} 00^{\prime}$ & $100^{\circ} 12^{\prime}$ & 04/10/1999 & 700 \\
\hline \multicolumn{5}{|l|}{ Cimar 6} \\
\hline E-5 & $33^{\circ} 00^{\prime}$ & $72^{\circ} 32^{\prime}$ & $27 / 09 / 2000$ & 1000 \\
\hline E-6 & $33^{\circ} 00^{\prime}$ & $73^{\circ} 31^{\prime}$ & $27 / 09 / 2000$ & 1000 \\
\hline E-13 & $33^{\circ} 00^{\prime}$ & $79^{\circ} 33^{\prime}$ & $02 / 10 / 2000$ & 1000 \\
\hline E-17 & $33^{\circ} 40^{\prime}$ & $80^{\circ} 52^{\prime}$ & $03 / 10 / 2000$ & 1000 \\
\hline E-43 & $31^{\circ} 47^{\prime}$ & $80^{\circ} 00^{\prime}$ & $04 / 10 / 2000$ & 1000 \\
\hline E-59 & $26^{\circ} 19^{\prime}$ & $80^{\circ} 02^{\prime}$ & $08 / 10 / 2000$ & 1000 \\
\hline E-65 & $26^{\circ} 59^{\prime}$ & $79^{\circ} 05^{\prime}$ & $09 / 10 / 2000$ & 1000 \\
\hline E-66 & $26^{\circ} 59^{\prime}$ & $78^{\circ} 18^{\prime}$ & $09 / 10 / 2000$ & 1000 \\
\hline E-69 & $26^{\circ} 59^{\prime}$ & $76^{\circ} 05^{\prime}$ & $10 / 10 / 2000$ & 1000 \\
\hline E-78 & $27^{\circ} 00^{\prime}$ & $70^{\circ} 52^{\prime}$ & $12 / 10 / 2000$ & 1000 \\
\hline
\end{tabular}

Quatrefages con una abundancia de 77,8\% (Tabla 2). En el crucero Cimar 6, se recolectaron sólo 19 ejemplares, destacándose nuevamente $T$. septentrionalis (42,1\%), seguido de Pelagobia longicirrata Greeff (Lopadorhynchidae) con un $21,1 \%$ (Tabla 3). Se documenta por primera vez la presencia, en aguas del Pacífico suroriental frente a Chile e islas oceánicas, de Lopadorhynchus uncinatus Fauvel, Pelagobia longicirrata Greeff, Vanadis minuta Treadwell, Vanadis crystallina Greeff y Watelio gravieri (Benham). Con estos resultados aumenta a 19 el número de especies de poliquetos pelágicos registradas frente a la costa de Chile e islas oceánicas.

\section{ALCIOPIDAE Ehlers}

\section{Torrea pelagica Chamberlin, 1919}

Torrea pelagica Chamberlin, 1919: 131-133, pl. 24, figs. 4-9; Rozbaczylo, 1985

Material examinado: Cimar 5: E-5 (MUAP 0032: 1 ejemplar), E-8 (MUAP 0033: 1 ejemplar), E-23
(MUAP 0034: 1 ejemplar), E-27 (MUAP 0035: 1 ejemplar), E-25 (MUAP 0036: 1 ejemplar); Cimar 6: E-59 (MUAP 0048: 1 ejemplar), E-65 (MUAP 0049: 1 ejemplar).

Caracterización: ejemplares incompletos; su longitud máxima es de 27,9 $\mathrm{mm}$ hasta el segmento parapodial 31; cuerpo delgado, translúcido; el prostomio presenta, en la parte media anterior, una protuberancia redondeada que puede estar retraída; de la superficie dorsal aparece un tentáculo medio delgado cónico; hacia adelante y los lados se ubican dos pares de antenas; probóscide delgada, con dos cuernos terminales y sin papilas entre ellos; primeros parápodos cortos, pero se incrementan regularmente en largo y grosor a medida que se avanza hacia el extremo posterior; los cirros neuropodiales de los somitos típicos son mucho más pequeños que los correspondientes cirros notopodiales; sin apéndice cirriforme en el ápice del lóbulo setígero; órganos segmentales de color oscuro; las setas son muy finas y transparentes, con piezas distales agudas y delgadas. 
Tabla 2. Especies de poliquetos pelágicos recolectadas durante el crucero Cimar 5. T: Tomopteridae, A: Alciopidae, L: Lopadorhynchidae, F: frecuencia de ocurrencia, AT: abundancia total y A: abundancia relativa (\%).

Table 2. Pelagic polychaetes species collected during the Cimar 5 cruise. T: Tomopteridae, A: Alciopidae, L: Lopadorhynchidae, F: frecuency of occurrence, AT: total abundance and A: relative abundance (\%).

\begin{tabular}{|c|c|c|c|c|c|c|c|c|c|c|c|c|c|}
\hline Especie & E-5 & E-8 & E-9 & E-16 & E-21 & E-23 & E-27 & E-30 & E-35 & E-43 & $\mathbf{F}$ & AT & $\mathbf{A}(\%)$ \\
\hline Tomopteris carpenterii $(\mathrm{T})$ & & & 1 & & & & & 1 & 1 & & 3 & 3 & 3,33 \\
\hline Tomopteris septentrionalis $(\mathrm{T})$ & 67 & & 1 & & & & 1 & & 1 & & 4 & 70 & 77,78 \\
\hline Torrea pelagica $(\mathrm{A})$ & 1 & 1 & & & & 1 & 1 & & 1 & & 5 & 5 & 5,56 \\
\hline Vanadis formosa (A) & 3 & & & 2 & & & & & 1 & & 3 & 6 & 6,67 \\
\hline Vanadis minuta $(\mathrm{A})$ & & & & & & & & & 1 & & 1 & 1 & 1,11 \\
\hline Vanadis longissima (A) & 2 & & & & & & & & & & 1 & 2 & 2,22 \\
\hline Watelio gravieri $(\mathrm{A})$ & & & & & 1 & & & & & & 1 & 1 & 1,11 \\
\hline Pelagobia longicirrata (L) & & & & & & & & & & 1 & 1 & 1 & 1,11 \\
\hline Lopadorhynchus uncinatus (L) & & & & & & & & & & 1 & 1 & 1 & 1,11 \\
\hline Total de ejemplares & & & & & & & & & & & & 90 & \\
\hline
\end{tabular}

Tabla 3. Especies de poliquetos pelágicos recolectadas durante el crucero Cimar 6. T: Tomopteridae, A: Alciopidae, L: Lopadorhynchidae, P: Polynoidae, F: frecuencia de ocurrencia, AT: abundancia total y A: abundancia relativa $(\%)$.

Table 3. Pelagic polychaetes species collected during the Cimar 6 cruise. T: Tomopteridae, A: Alciopidae, L: Lopadorhynchidae, P: Polynoidae, F: frecuency of occurrence, AT: total abundance and A: relative abundance $(\%)$.

\begin{tabular}{|c|c|c|c|c|c|c|c|c|c|c|c|c|c|c|}
\hline Especie & E-5 & E-6 & E-13 & E-17 & 7 E-43 & 3 E-55 & E-59 & E-65 & E-66 & E-69 & E-78 & $\mathbf{F}$ & AT & $\mathrm{A}(\%)$ \\
\hline Enapteris euchaeta $(\mathrm{T})$ & 1 & & & & & & & & & & & 1 & 1 & 5,26 \\
\hline Tomopteris carpenterii $(\mathrm{T})$ & & & & & 1 & 1 & & & & & & 1 & 1 & 5,26 \\
\hline Tomopteris septentrionalis $(\mathrm{T})$ & 4 & & & 1 & & 1 & & 1 & & & 1 & 5 & 8 & 42,11 \\
\hline Vanadis crystallina $(\mathrm{A})$ & & & & & & & & & & 1 & & 1 & 1 & 5,26 \\
\hline Torrea pelagica (A) & & & & & & & 1 & 1 & & & & 2 & 2 & 10,53 \\
\hline Pelagobia longicirrata (L) & & & 1 & & & & 3 & & & & & 2 & 4 & 21,05 \\
\hline Drieschia nans $(\mathrm{P})$ & & & & & & & & & 1 & & 1 & 2 & 2 & 10,53 \\
\hline Total de ejemplares & & & & & & & & & & & & & 15 & \\
\hline
\end{tabular}

Distribución geográfica: entre Callao, Perú e isla de Pascua $\left(17^{\circ} 55^{\prime} \mathrm{S}, 87^{\circ} 42^{\prime} \mathrm{W}\right)$ y entre isla de Pascua e islas Galápagos $\left(20^{\circ} 29^{\prime} \mathrm{S}, 103^{\circ} 26^{\prime} \mathrm{W}\right)$ (Chamberlin, 1919); en Chile, frente a Caldera y Valparaíso (este estudio).

\section{Vanadis crystallina Greeff, 1876}

Vanadis crystallina Greeff, 1876: 68-69, pl. 4, figs. 35-39; Apstein, 1900: 10-11, pl. 1, fig. 7; Fauvel, 1923: 206-207, fig. 77d-e; Monro, 1936: 116-117; Dales, 1957: 118-119, figs. 25-27; Tebble, 1960: 186-187.

Material examinado: Cimar 6: E-69 (MUAP 0047: 1 ejemplar).
Caracterización: ejemplar incompleto; midió 13 $\mathrm{mm}$ hasta el segmento parapodial 15; con dos pares de antenas frontales pequeñas (las más ventrales son las más largas), y una antena corta digitiforme, difícil de ver; tres pares de cirros tentaculares, siendo los más posteriores los más pequeños; probóscide larga, con dos largos cuernos terminales y entre ellos cuatro a seis papilas trilobuladas; los primeros siete a diez pares de parápodos son rudimentarios, y hacia el extremo posterior están desarrollados, con un gran cirro dorsal foliáceo y uno ventral más pequeño; con un apéndice cirriforme relativamente largo en el ápice del lóbulo setígero y órganos segmentales pigmentados a partir del segmento parapodial 7; el 
ejemplar observado es hembra pues presenta los cirros dorsales de los dos primeros pares de parápodos modificados como receptáculos seminales.

Observaciones: según Dales (1957), V. crystallina y $V$. minuta pueden ser confundidas y las compara señalando varias características contrastantes. De acuerdo con Dales (1957), el único registro previo que había en el Pacífico sería el de un espécimen descrito por Treadwell (1928) como V. collata, recolectado durante la expedición Arcturus, en la región de las islas Galápagos ( $\left.2^{\circ} 0^{\prime} \mathrm{S}, 89^{\circ} 30^{\prime} \mathrm{W}\right)$.

Distribución geográfica: en Chile, frente a isla San Félix (este estudio); en el Atlántico sur hasta el límite sur de la Convergencia Subtropical (Tebble, 1960).

\section{Vanadis formosa Claparède, 1870}

Vanadis formosa Claparède, 1870: 480-482, pl. 10, fig. 3; Chamberlin, 1919: 134-135; Fauvel, 1923: 205-206, fig. 77 a-c; Støp-Bowitz, 1948: 25-26, fig. 17; Tebble, 1960: 185-186; Day, 1967: 181, fig. 7.2.a-e; Rozbaczylo, 1985: 58.

Vanadis latocirrata Apstein, 1891: 7-8, figs. 9-11.

Material examinado: Cimar 5: E-5 (MUAP 0027: 3 ejemplares), E-16 (MUAP 0028: 2 ejemplares), E-35 (MUAP 0029: 1 ejemplar).

Caracterización: los ejemplares están incompletos; su longitud máxima es de $12 \mathrm{~mm}$ desde la parte anterior del prostomio hasta el parápodo 27; antenas frontales superiores cortas, y las del par inferior más largas; la antena media es digitiforme; ojos laterales; probóscide larga, con un par de cuernos laterales y papilas trilobuladas entre ambos; tres pares de cirros tentaculares ordenados $1+1+1$; el primero se encuentra montado sobre un cirróforo abultado adherido a la superficie inferior del ojo, es ligeramente más largo que los otros; el cuarto y quinto segmento sin lóbulos setígeros y en las hembras los cirros dorsales de ambos se alargan para actuar como receptáculos seminales; el primer lóbulo setígero y setas están en el segmento 6; los primeros parápodos son pequeños, pero el lóbulo setígero y el cirro ventral miden más de la mitad de largo que el cirro dorsal; los parápodos alcanzan su máximo desarrollo alrededor del segmento 12 con cirro dorsal foliáceo y aguzado; el lóbulo setígero con un apéndice cirriforme largo y un cirro ventral foliáceo ligeramente más pequeño que el dorsal; órganos segmentales como cojinetes ovales detrás de los parápodos y pigmentados a partir del segundo setígero (segmento 7) hacia la parte posterior.

Distribución geográfica: costa de Chile (Apstein, 1891), a 1.155 millas frente a río Camarones, entre Arica y Pisagua, a 1.425 millas frente a Tocopilla y a 336 millas al NE de isla de Pascua (Chamberlin, 1919), frente a Caldera e isla de Pascua (este estudio); frente a Perú, entre Perú e isla de Pascua, entre isla de Pascua e islas Galápagos, entre islas Galápagos e islas Paumotu, frente a islas Galápagos (Chamberlin, 1919); mar Mediterráneo y aguas cálidas de los océanos Atlántico y Pacífico (Day, 1967).

\section{Vanadis longissima (Levinsen, 1885)}

Rhynchonerella longissima Levinsen, 1885: 330, figs. 7-10.

Vanadis fasciata Apstein, 1890: 543, pl. 38, figs. 19; 1891: 130.

Vanadis longissima: Apstein, 1900: 1, pl. 1, figs. 89; Fauvel, 1923: 207, fig. 77 f-g; Monro, 1930: 7981, fig. 24; Dales, 1957: 121, figs. 31-33; Tebble, 1962: 392, figs. 8 y 38; Hartman, 1964: 63, pl. 18, figs. 8-9; Day, 1967: 186, fig. 7.3.a-c; Rozbaczylo, 1985: 58-59.

Material examinado: Cimar 5: E-5 (MUAP 0030: 2 ejemplares).

Caracterización: los ejemplares están incompletos; su longitud máxima es de 3,9 mm hasta el parápodo 17; antena media digitiforme al igual que las frontales; prostomio con ojos dirigidos lateroventralmente; probóscide sin cuernos laterales, pero con aproximadamente 12 papilas marginales; tres pares de cirros tentaculares sobre los tres primeros segmentos ordenados $1+1+1$; los del tercer par son los más cortos; los parápodos de los segmentos 4-8 están reducidos y carecen de lóbulos setígeros y de setas, las que aparecen por primera vez en los segmentos 7-9; los parápodos completamente desarrollados están constituidos por un cirro dorsal lamelar más largo que ancho, un lóbulo setígero largo terminado en un apéndice cirriforme y un cirro ventral más pequeño, pero similar al dorsal; los ejemplares observados son machos pues presentan órganos segmentales con una fuerte pigmentación café, dispuestos en bandas anchas, cada 4-10 segmentos (en las hembras los cirros dorsales de los segmentos 4 y 5 se presentan dilatados para actuar como receptáculos seminales). Todas las setas son finas espinígeras compuestas.

Distribución geográfica: en Chile, frente a Calde- 
ra (este estudio); Paso Drake, al sur de las islas Hermite (Monro, 1930); en aguas tropicales y subtropicales del Pacífico norte y Atlántico (Orensanz \& Ramírez, 1973).

\section{Vanadis minuta Treadwell, 1906}

Vanadis minuta Treadwell, 1906: 1158, figs. 25-27; Dales, 1957: 119-121, figs. 29-30; Tebble, 1962: 390, 440, figs. 7 y 37; Day, 1967: 184-185, fig. 7.2. k-m; Orensanz \& Ramírez, 1973: 40-42, pl. 6, figs. 1-5.

Material examinado: Cimar 5: E-35 (MUAP 0031: 1 ejemplar).

Caracterización: ejemplar incompleto; su longitud es de $8,1 \mathrm{~mm}$ desde la parte anterior del prostomio hasta el parápodo 19; antena media reducida a una simple cresta ubicada entre los ojos; antenas frontoventrales dirigidas hacia abajo y más largas que las dorsales; ojos dirigidos anterolateralmente; probóscide con un par de largos cuernos laterales, sin papilas entre ellos; tres pares de cirros tentaculares sobre los tres primeros segmentos; los parápodos de los segmentos $4^{\circ}-7^{\circ}-9^{\circ}$ están reducidos, sin lóbulos setígeros ni setas, cada uno con un cirro dorsal lanceolado y un cirro ventral diminuto, globular; en los machos, los cirros dorsales de los segmentos $4^{\circ}-5^{\circ}$ no son más largos que los de los segmentos siguientes; en las hembras, los cirros dorsales del quinto segmento se modifican para formar receptáculos seminales; parápodos normales con un lóbulo setígero que termina en un largo apéndice cirriforme, un cirro dorsal oval-lanceolado más largo que ancho y un cirro ventral lamelar; órganos segmentales incoloros o ligeramente coloreados a partir del segmento 12, aproximadamente; todas las setas son delgadas espinígeras compuestas.

Distribución geográfica: en aguas del Pacífico y región de las islas Hawaii (Dales, 1957); en Chile, alrededor de isla de Pascua (este estudio).

\section{Watelio gravieri (Benham, 1929)}

Callizona gravieri Benham, 1929: 190.

Watelio longifoliata Støp-Bowitz, 1948: 37, fig. 23 a-d.

Watelio gravieri: Dales, 1957: 134-135, figs. 47-50.

Material examinado: Cimar 5: E-21 (MUAP 0037: 1 ejemplar).

Caracterización: ejemplar incompleto; su longitud es de $29 \mathrm{~mm}$ hasta el segmento parapodial 63; cuer- po alargado, transparente, un poco aplanado dorsoventralmente; probóscide provista de papilas lanceoladas, similares, alrededor del margen; prostomio proyectado al frente de los ojos, con dos pares de antenas frontales muy pequeñas y una antena media pequeña entre los ojos; cuatro pares de cirros tentaculares; segmentos siguientes con el cirro parapodial ventral excepcionalmente más largo que el cirro dorsal; lóbulo setígero con un apéndice corto, romo, ubicado dorsalmente a la acícula que es extremadamente larga, robusta, que se proyecta desde la punta del lóbulo setígero a una distancia igual al largo del lóbulo mismo; con numerosas setas, principalmente largas, delgadas y compuestas, pero también algunas setas simples delgadas se presentan en la parte ventral del manojo; órganos segmentales obscuros y pequeños, con una gran proyección cónica sobresaliente en el lado ventral cerca de la base de cada parápodo.

Distribución geográfica: en Chile, alrededor de isla de Pascua (este estudio); Nueva Zelanda, Atlántico norte y sur de las Azores (Dales, 1957).

\section{LOPADORHYNCHIDAE Claparède}

\section{Lopadorhynchus uncinatus Fauvel, 1915}

Lopadorhynchus uncinatus Fauvel, 1915: 3, fig. 2; 1916: 57, pl. 1, figs. 2-3, pl. 4, figs. 4-14; 1923 : 184, figs. 67a-g; 1932: 75; Monro, 1936: 113; StøpBowitz, 1948: 17-18, fig. 11; Dales, 1957: 101-104, figs. 1-5; Tebble, 1960: 201, 259-261, text-fig. 52.

Lopadorhynchus varius Treadwell, 1943: 32, pl. 1, figs. 7-10.

Material examinado: Cimar 5: E-43 (MUAP 0039: 1 ejemplar).

Caracterización: el ejemplar mide 7,4 mm de longitud y $2,8 \mathrm{~mm}$ de ancho máximo a la altura del parápodo 8; prostomio ancho y extendido lateralmente, con dos pares de antenas; con dos ojos; tres pares de cirros tentaculares, los dos pares más anteriores son de igual longitud y se proyectan más alla de las puntas de las antenas; el tercer par surge ventralmente y es mucho más corto que los otros; los primeros dos segmentos setígeros son más prominentes que el resto, grandes, robustos, y direccionados lateralmente, con cuatro a seis ganchos gruesos curvos, unidentados, rodeados en la base por una expansión membranosa del lóbulo setígero, como un collar, y con un cirro dorsal pe- 
queño, sin cirro ventral; el tercer setígero y los siguientes con setas compuestas y con cirros dorsal y ventral.

Distribución geográfica: en Chile, alrededor de isla de Pascua (este estudio); en el Pacífico norte y sur, Atlántico norte y sur, y océano Índico (Dales, 1957).

\section{Pelagobia longicirrata Greeff, 1879}

Pelagobia longicirrata Greeff, 1879: 247-249, pl. 14. figs. 23-25; Augener, 1929: 291; Fauvel, 1936: 16-17; Støp-Bowitz, 1949: 4; 1951: 6; Dales, 1957: 107-108, figs. 11-13; Tebble, 1962: 419-420, fig. 22; Hartman, 1964: 64, pl. 19, figs. 5-6; Day, 1967: 163, fig. 5.7. f-i; Orensanz \& Ramírez, 1973: 2527, pl. 2, figs. 1-5; Rozbaczylo, 1985: 60; Knox \& Cameron, 1998: 43.

Material examinado: Cimar 5: E-43 (MUAP 0038: 1 ejemplar); Cimar 6: E-13 (MUAP 0050: 1 ejemplar), E-59 (MUAP 0051: 3 ejemplares).

Caracterización: ejemplares incompletos; su longitud máxima es de $3 \mathrm{~mm}$ desde la parte anterior del prostomio hasta el parápodo 10; prostomio subcónico o truncado anteriormente, con un par de ojos pequeños y dos pares de antenas delgadas; segmento tentacular con dos pares de cirros tentaculares largos, subiguales, más largos que el ancho del cuerpo y algunas setas; segundo segmento setígero sin cirros dorsales; parápodos del resto del cuerpo con un lóbulo setígero cónico sostenido por una acícula gruesa y cirros dorsales y ventrales delgados, subiguales, distalmente afinados y más largos que el lóbulo setígero; setas compuestas, fuertemente heterogonfas, con la pieza distal aguzada, delgada, dentada a lo largo de uno de sus bordes.

Distribución geográfica: cosmopolita; islas Shetland del Sur, norte de isla Rey Jorge (StøpBowitz, 1951); en Chile, alrededor de las islas San Félix y Robinson Crusoe (este estudio); en el Atlántico, desde Groenlandia hasta la Antártica; en el Mediterráneo e Índico (Dales, 1957).

\section{POLYNOIDAE Malmgren}

\section{Drieschia nans (Chamberlin, 1919)}

Plotolepis nans Chamberlin, 1919: 41-44, pl. 7, figs. 3-4.

Material examinado: Cimar 6: E-66 (MUAP 0052: 1 ejemplar), E-78 (MUAP 0053: 1 ejemplar).
Caracterización: los dos ejemplares están incompletos; la longitud máxima del más largo es de 8,6 $\mathrm{mm}$ desde la parte anterior del prostomio hasta el parápodo 13 y su ancho máximo de $6,1 \mathrm{~mm}$, a la altura del parápodo 10; los élitros de ambos ejemplares están ausentes; el cuerpo y sus apéndices son incoloros, translúcidos; prostomio levemente más largo que ancho, con una escisión media anterior en la que se encuentra el cirróforo de la antena media y a cada lado se encuentran las antenas laterales; posteriormente presenta una lengüeta triangular en cuyo interior se proyecta la parte anterior del segundo segmento; con dos pares de ojos pequeños, los del par anterior más separados y los de cada lado más próximos entre sí; dos pares de cirros tentaculares, los dorsales y ventrales de igual longitud; los parápodos son largos y relativamente delgados, sin lóbulo notopodial ni setas notopodiales; neurópodos alargados, con numerosas setas dispuestas como un abanico, en dos grupos, uno ventral con 2 a 5 setas más gruesas y cortas y uno dorsal con setas más numerosas, largas y delgadas; los cirros notopodiales se encuentran unidos en el extremo basal sobre los parápodos; sus cirróforos son notoriamente grandes, inflados, subcilíndricos; los cirrostilos son notoriamente largos; el quinto par de notocirros son marcadamente diferentes de los demás.

Distribución geográfica: en Chile, frente a Caldera e isla San Félix (este estudio); isla de Pascua, 29 millas al NE de Cabo Norte (Chamberlin, 1919).

\section{TOMOPTERIDAE Grube}

\section{Enapteris euchaeta (Chun, 1888)}

Tomopteris euchaeta Chun, 1888: 19, pl. 3, figs. 13, 6-9; Day, 1967: 202, fig. 8.2.a-b; Fernández-Álamo, 2000: 46-47.

Tomopteris nisseni Rosa, 1908a: 1; Støp-Bowitz, 1948: 44, fig. 29 (adulto).

Enapteris euchaeta: Rosa, 1908b: 269-272; Fauvel, 1923: 218, fig. 82 a-c (juvenil); Orensanz \& Ramírez, 1973: 58-60, pl. 13, figs. 1-4; Rozbaczylo et al., 1987: 103, fig. 2a.

Material examinado: Cimar 6: E-6 (MUAP 0040: 1 ejemplar).

Caracterización: el ejemplar completo midió 47,9 $\mathrm{mm}$ de longitud total y $10,5 \mathrm{~mm}$ de ancho máximo a la altura del parápodo 6; hasta 39 pares de 
parápodos desarrollados y una región caudal provista de parápodos rudimentarios carentes de pínulas; prostomio hendido frontalmente, con un par de antenas dirigidas posterolateralmente; el primer par de cirros está ausente; el segundo par de cirros mide aproximadamente 2/3 la longitud del cuerpo; cada rama parapodial está rodeada, en su borde externo, por pínulas anchas más o menos ovaladas que no alcanzan el margen interno de las ramas; las ramas parapodiales son relativamente cortas, sus extremos terminales están expandidos y no se aprecian claramente definidos; las glándulas cromófilas están presentes a partir del cuarto par de parápodos, son voluminosas y sobresalen de las pínulas, se sitúan en la parte ventro-apical de las pínulas ventrales; con glándulas hialinas en la pínula ventral, en posición apical, a partir del tercer par de parápodos.

Observaciones: Enapteris Rosa se diferencia de Tomopteris Eschscholtz, por la forma de las ramas parapodiales y la extensión de las pínulas. En Tomopteris las ramas parapodiales son cónicas y las pínulas rodean completamente a las ramas, mientras que en Enapteris las ramas parapodiales son rectangulares y las pínulas no bordean el lado interno de las mismas. Day (1967), indica que estas diferencias se deberían a que Rosa (1908b) analizó ejemplares de estadios juveniles, por esta razón no se aceptaría la validez de Enapteris y sería considerado como sinónimo de Tomopteris (Rouse \& Pleijel, 2001). Fernández-Álamo (2000) discute que el carácter principal usado por Rosa (1908b) no es correcto, ya que las pínulas se extenderían también por la parte interna, sólo que en esta región son hialinas. De acuerdo con Orensanz \& Ramírez (1973) y Rozbaczylo et al. (1987), se considera a Enapteris como un género válido pues las características morfológicas exhibidas por el ejemplar adulto examinado concuerdan con los caracteres que definen al género.

Distribución geográfica: en Chile, frente a Coquimbo y Valparaíso (este estudio), mar Mediterráneo, océanos Atlántico, Pacífico norte e Índico (Day, 1967). En el Pacífico tropical oriental (Fernández-Álamo, 2000).

\section{Tomopteris carpenterii Quatrefages, 1865}

Tomopteris carpenterii Quatrefages, 1865: 227-228, pl. 20, figs. 1-2; Ehlers, 1917: 229-231, pl. 12, figs. 9-12; Benham, 1921: 61, pl. 8, figs. 64-66; Monro, 1936: 120-121; Støp-Bowitz, 1949: 16-17, figs. 6-
7; Tebble, 1960: 174, fig. 7; Hartman, 1964: 67-69, pl. 21, fig. 1; Rozbaczylo et al., 1987: 106-107, fig. 2c; Knox \& Cameron, 1998: 45.

Material examinado: Cimar 5: E-9 (MUAP 0020: 1 ejemplar), E-30 (MUAP 0021: 1 ejemplar), E-35 (MUAP 0022: 1 ejemplar); Cimar 6: E-43 ( MUAP 0041: 1 ejemplar).

Caracterización: los ejemplares analizados midieron entre 7,6 y 54,4 mm de longitud total y entre 2,5 y $13 \mathrm{~mm}$ de ancho máximo a nivel de los parápodos 6 y 9; sin región caudal; prostomio hendido frontalmente, con antenas dirigidas posterolateralmente; el primer par de cirros está ausente, el segundo par mide aproximadamente 2/3 la longitud del cuerpo; las glándulas cromófilas están presentes a partir del cuarto par de parápodos hasta el último; son cupuliformes y están muy desarrolladas en los parápodos 4 y 5; las glándulas hialinas son muy notorias y están fuertemente pigmentadas a partir del tercer par de parápodos en el extremo de la rama ventral.

Distribución geográfica: regiones Antártica y Subantártica; dentro de los límites del territorio antártico chileno $\left(53^{\circ}\right.$ a $\left.90^{\circ} \mathrm{W}\right)$ ha sido recolectada al NNE de la isla Pedro I y al frente del Estrecho Matha (Monro, 1936; Støp-Bowitz, 1949). Según Tebble (1960), T. carpenterii sería endémica de aguas antárticas, no sobrepasando al norte de la Convergencia Antártica. En este estudio, se registró en aguas del Pacífico suroriental frente a Caldera e islas de Pascua y Robinson Crusoe.

\section{Tomopteris septentrionalis Quatrefages, 1865}

Tomopteris septentrionalis Quatrefages, 1865: 229; Rosa, 1908b: 297-301, fig. 17; Gravier, 1911: 3; Benham, 1929: 195; Monro, 1936: 121-122; Dales, 1957: 145, figs. 51f, 52g; Støp-Bowitz, 1949: 12; Tebble, 1962: 382-383, fig. 5; Day, 1967: 205-206, figs. 8.2.1-m; Orensanz \& Ramírez, 1973: 65-67, pl. 16, figs. 1-4; Rozbaczylo et al., 1987: 108-110, fig. 2f; Knox \& Cameron, 1998: 45-46.

Material examinado: Cimar 5: E-5 (MUAP 0023: 67 ejemplares), E-9 (MUAP 0024: 1 ejemplar), E27 (MUAP 0025: 1 ejemplar), E-35 (MUAP 0026: 1 ejemplar); Cimar 6: E-5 (MUAP 0042: 4 ejemplares), E-17 (MUAP 0043: 1 ejemplar), E-43 (MUAP 0044: 1 ejemplar), E-65 (MUAP 0045: 1 ejemplar), E-78 (MUAP 0046: 1 ejemplar).

Caracterización: los ejemplares examinados mi- 
dieron entre 2,9 y $20,9 \mathrm{~mm}$ de longitud total y entre 0,9 y $5,4 \mathrm{~mm}$ de ancho máximo a la altura de los parápodos 5 y 6 ; con 20-24 pares de parápodos, sin región caudal; prostomio hendido frontalmente; con dos ojos; el primer par de cirros está ausente; el segundo par de cirros mide aproximadamente 3/4 la longitud del cuerpo; las pínulas son ovales; a contar del $4^{\circ}$ par de parápodos con glándulas cromófilas en forma de numerosos túbulos alargados que se abren independientemente a lo largo de los bordes ventral y apical de la pínula ventral; las glándulas hialinas, aunque difíciles de observar, están ubicadas en la parte apical de las pínulas ventrales.

Observaciones: según Tebble (1960), la presencia de la glándula cromófila difusa en $T$. septentrionalis ha sido causa de confusión con T. planktonis Apstein, sin embargo, T. planktonis presenta una glándula cromófila compacta en los adultos y sólo en ejemplares juveniles existe esta similitud con $T$. septentrionalis.

Distribución geográfica: cosmopolita; en Chile se distribuye desde Iquique hasta frente a la desembocadura del río Mataquito (3506'S, 73³8'W) (Rosa, 1908b); alrededor de isla de Pascua, islas Desventuradas y archipiélago de Juan Fernández (este estudio); frente a bahía Misaki, Japón (Isuka, 1914); mar de Okhotsk, mar de Bering, zonas subtropical y subártica (Uschakov, 1955, 1957 fide Tebble, 1962); golfo de Alaska (Berkeley \& Berkeley, 1957); frente a la Columbia Británica (Berkeley, 1924, Berkeley \& Berkeley, 1948); desde cabo Disappointment, Oregon, al sur de la bahía de San Francisco (Dales, 1957); en aguas tropicales, subtropicales y subárticas (Treadwell, 1943); en el océano Atlántico desde Groenlandia hasta la Antártica; en el mar del Norte y Mediterráneo; en el Atlántico sur frente a las costas de África del sur (Day, 1967) y en el Atlántico sudoeste en aguas del extremo sur de la plataforma brasileña, uruguaya y a lo largo de la plataforma argentina $\left(30^{\circ}-52^{\circ} \mathrm{S}\right)$ (Orensanz \& Ramírez, 1973). En algunas localidades ubicadas en el Pacífico tropical oriental (Fernández-Álamo, 2000).

\section{DISCUSIÓN}

Los resultados obtenidos durante las campañas científicas Cimar 5 y Cimar 6 constituyen un importante aporte al conocimiento taxonómico de los poliquetos pelágicos del Pacífico suroriental frente a Chile e islas oceánicas, permitiendo configurar preliminarmente una visión acerca de la naturaleza y magnitud de la diversidad del grupo en esta área del Pacífico sur.

Como resultado de su inusual morfología, las relaciones filogenéticas entre las familias de poliquetos pelágicos no son claras, lo que ha llevado a que sus integrantes sean considerados, en general, como organismos aberrantes (Pettibone, 1982), dificultando su reunión en grupos o clados (Rouse \& Fauchald, 1997; Rouse \& Pleijel, 2001). Por otra parte, para algunas familias su condición de pelágicas aún no está claramente establecida; por ejemplo, Fernández-Álamo \& Thuesen (1999) reconocen en aguas del Pacífico noroeste y aguas ecuatoriales frente a la costa de África (Golfo de Guinea) a las familias Flotidae Hartman e Yndolaciidae Støp-Bowitz, respectivamente, ambas integradas por una sola especie. Según Rouse \& Pleijel (2001), Flota Hartman está relacionado a Poeobiidae Heath en los clados Palpata, Canalipalpata, Terebellida, Cirratuliformia, en tanto que Yndolacia es ubicada en Phyllodocida incertae sedis. En cuanto a las familias Tomopteridae, Alciopidae y Lopadorhynchidae, representadas frente a Chile e islas océanicas, Rouse \& Pleijel (2001) reconocen su afinidad con los clados Palpata, Aciculata, Phyllodocida "unplaced", y consideran a Alciopidae como sinónimo de Phyllodocidae denominándolo grupo "Alciopini”. Estas propuestas filogenéticas resultan aún un escenario bastante complejo de interpretar y en muchos casos, aún existen taxa con situaciones no resueltas, lo que obscurece aún más el panorama de una configuración general acerca de las probables relaciones entre las familias pelágicas

Las transectas efectuadas en ambos cruceros permitieron comparar parte del sistema de corrientes de Humboldt y aguas oceánicas alrededor de las islas de Pascua, San Félix, San Ambrosio y archipiélago de Juan Fernández, encontrándose nuevos registros de taxa de poliquetos pelágicos para esta área del Pacífico suroriental, lo que en términos de afinidades zoogeográficas permitió definir que mayoritariamente estos taxa tendrían un patrón de distribución cosmopolita, a excepción de Torrea pelagica y Drieschia nans que serían endémicas para el Pacífico sur.

Los cruceros de investigación marina Cimar 5 y Cimar 6 han permitido obtener información relevante de tipo cualitativa sobre la biodiversidad de poliquetos pelágicos en aguas del Pacífico 
suroriental frente a Chile e islas oceánicas. Sin embargo, el arte de muestreo (Red IKMT) empleado en su recolección, lamentablemente no permite obtener información sobre la distribución estratificada del plancton en la columna de agua, lo que a su vez impide una comparación multifactorial, y la obtención de antecedentes biológicos y ecológicos de las especies, en términos cuantitativos. A pesar que los poliquetos pelágicos conforman un grupo de escasa representación en el zooplancton, en cuanto a número de especies y abundancia relativa, y dada su pequeña talla, con escasa significación en la biomasa total de las comunidades zooplanctónicas (Orensanz \& Ramírez, 1973), se reconoce su importancia en las cadenas tróficas de los océanos del mundo, debido a que algunas especies son voraces depredadores en el plancton y a su vez son fuente de alimento para varios tipos de peces (Pettibone, 1963, Fernández-Álamo, 2000). Por estos motivos, resulta necesario intensificar los muestreos de tipo cuantitativo estratificado de plancton para incrementar el conocimiento sobre estos temas, permitiendo establecer desde una perspectiva funcional sus relaciones en la dinámica oceánica del plancton, en términos de conexiones tróficas, asociaciones a masas de aguas específicas (i.e. especies indicadoras) y patrones de migraciones nictimerales en la columna de agua frente a la costa del Pacífico suroriental de Chile e islas oceánicas.

\section{AGRADECIMIENTOS}

Al Comité Oceanográfico Nacional por el apoyo logístico prestado para la realización de los cruceros Cimar 5 y Cimar 6. Al Prof. Walter Sielfeld de la Universidad Arturo Prat, investigador principal del Proyecto Fauna Mesopelágica, por sus comentarios y apoyo. A dos revisores anónimos por sus valiosas sugerencias y comentarios críticos.

\section{REFERENCIAS}

Apstein, C. 1890. Vanadis fasciata, eine neue Alciopide. Zool. Jb., 5: 543-545.

Apstein, C. 1891. Die Alciopiden des naturhistorischen Museums in Hamburg. Jahrb. Hamburg wiss. Anst., 8: 3-19.

Apstein, C. 1900. Die Alciopiden und Tomopteriden der Plankton-Expedition. Ergebn. der PlanktonExped. Der Humboldt-Stiftung, II(b): 1-61.

Augener, H. 1929. Beiträge zur Planktonbevölkerung der Weddellsee. Int. Revue d. ges. Hydrob. u. Hydrog., 22: 273-312.

Benham, W.B. 1921. Polychaeta. Scient. Rep. Australas. Antarct. Exped. 1911-1914, ser. C. Zool. Bot. 6(3):1-128.

Benham, W.B. 1929. The pelagic Polychaeta. British Antarctic ("Terra Nova") Expedition, 1910. Nat. Hist. Rep., Zool., 7(3): 183-201.

Berkeley, E. 1924. Polychaetous Annelids from the Nanaimo District. 2. Phyllodocidae to Nereidae. Contr. Can. Biol. Fish., New Ser., 2: 287-294.

Berkeley, E. \& C. Berkeley. 1948. Annelida, Polychaeta errantia. Fish. Res. Bd. Can., Can. Pac. Fauna, 9b(1): 1-100.

Berkeley, E. \& C. Berkeley. 1957. On some pelagic polychaeta from the Northeast Pacific north of latitude $40^{\circ} \mathrm{N}$ and east of longitude $175^{\circ} \mathrm{W}$. Can. J. Zool., 35: 573-578.

Chamberlin, R.V. 1919. The Annelida Polychaeta. Mem. Mus. Comp. Zool. Harvard, 48: 1-154.

Chun, C. 1888. Die pelagische Thierwelt in grösseren Meerestiefen und ihre Bezichungen zu der Oberflächenfauna. Bibliotheca zoologica Caasel, 1: 1-66.

Claparède, E. 1870. Les annélides chétopodes du Golfe de Naples. Mém. Soc. Phys. Hist. Nat., Genéve, 20(2): 365-542.

Dales, R.P. 1957. Pelagic polychaetes of the Pacific Ocean. Bull. Scripps Inst. Oceanogr., 7(2): 99-167.

Day, J.H. 1967. A monograph on the Polychaeta of Southern Africa. Part I. Errantia. Brit. Mus. Nat. Hist. Publ., 656: 1- 458.

Ehlers, E. 1917. Die Gymnocopa der deutschen Südpolar Expedition 1901-1903. Deutsch Südpolar-Expedition, 16(3): 221-233.

Fauchald, K. 1977. The polychaete worms: Definitions and keys to the Orders, Families and Genera. Nat. Hist. Mus. Los Angeles County. Sci. Ser., 28: 1-190.

Fauvel, P. 1915. Polychètes pélagiques nouvelles des campagnes de la Princesse-Alice (note 
préliminare). Bull. Inst. Océanogr. Monaco, 305 : $1-11$.

Fauvel, P. 1916. Annélides polychètes pélagiques prevenant des campagnes de l'Hirondelle et la Princesse-Alice (1885-1910). Res. Camp. Sci. Monaco, 48: 1-152.

Fauvel, P. 1923. Polychètes errantes. Faune Fr., 5: 1448.

Fauvel, P. 1932. Annélides polychètes prevenant des campagnes de 1'Hirondelle II (1911-1915). Rés. Camp. Sci. Monaco, 85: 1-50.

Fauvel, P. 1936. Polychètes. Expédition Antarctique Belge. Resultats du voyage de la Belgica en 189799, 1-46.

Fernández-Álamo, M.A. 2000. Tomopterids (Annelida: Polychaeta) from the Eastern Tropical Pacific Ocean. Bull. Mar. Sci., 67(1): 45-53.

Fernández-Álamo, M.A. \& E. Thuesen. 1999. Polychaeta. En: D. Bolstovkoy (ed.). South Atlantic Zooplankton. Backhyus Publishers, Leiden. pp. 595-619.

Gravier, C. 1911. Annélides polychétes recuielles par la Seconde Expédition Antarctique Française (1908-1910). Deuxième Expedition Antarctique Française, 1: 1-165.

Greeff, R. 1876. Untersuchungen über Alciopiden. Nova Acta Leopold-Carolin. d. Akad. Naturfor. Dresden, 39: 33-132.

Greeff, R. 1879. Ueber pelagische Anneliden von der Küste der Canarischen Inseln. Z. wiss. Zool., 32: 237-284.

Hartman, O. 1964. Polychaeta Errantia of Antarctica. Antarc. Res. Ser., 3:1-131.

Hutchings, P. 2000. Family Poeobiidae. En: P.L. Beesley, G.J.B. Ross \& C.J. Glasby (eds.). Polychaetes and allies: The southern synthesis. Fauna of Australia. Vol. 4A. Polychaeta, Myzostomida, Pogonophora, Echiura, Sipuncula. CSIRO Publishing, Melbourne, pp. 222-224.

Izuka, A. 1914. On the pelagic Annelids of Japan. J. Coll. Sci., 36: 1-14.

Knox, G.A. \& D.B. Cameron. 1998. The marine fauna of the Ross Sea: Polychaeta. NIWA Biodiversity Memoir, 108: 1-125.
Levinsen, G.M.R. 1885. Spolia Atlantica. Om nogle pelagiske Annulata. Skr. Vidensk. Selsk. Kbh. (Nat. Math.), 3(2): 321-344.

Monro, C.C.A. 1930. Polychaete worms. Discovery Rep., 2: 1-222.

Monro, C.C.A. 1936. Polychaete worms. II. Discovery Rep., 12: 59-198.

Orensanz, J.M. \& F.C. Ramírez. 1973. Taxonomía y distribución de los poliquetos pelágicos del Atlántico Sudoccidental. Bol. Inst. Biol. Mar., Mar del Plata, 21: 1-122.

Pettibone, M. 1963. Marine polychaete worms of the New England region. 1. Families Aphroditidae through Trocochaetidae. Bull. U.S. Nat. Mus., 227: $1-356$.

Pettibone, M. 1982. Annelida. En: S.B. Parker (ed.). Synopsis and classification of living organism. McGraw Hill, New York, pp. 1-43.

Quatrefages, A. de. 1865. Historie naturelle des Annéles marins et d'eau douce. Annélides et Géphryriens. Paris, Libraire Encyclopédique de Roret, 2(3): 1-336.

Rosa, D. 1908a. Nuove specie di tomopteridi. Diagnosi preliminari. Bull. Mus. Zool. Anat. Comp. Torino, 23: 1 .

Rosa, D. 1908b. Raccolte planctoniche fatte dalla R. Nave Liguria nel viaggio di circonnavigazione del 1903-1905 sotto il commando di S.A.R. Luigi di Savoia, duca degli Abruzzi. Anellidi, pt. I. Tomopteridi. Publ. Ist. Studi sup. prat. Firenze, Sezione di scienze fisiche e naturali, 1(5): 245-327.

Rouse, G. \& K. Fauchald. 1997. Cladistics and polychaetes. Zool. Scr., 26(2): 139-204.

Rouse, G. \& F. Pleijel. 2001. Polychaetes. Oxford University Press, Oxford, 1-354.

Rozbaczylo, N. 1985. Los anélidos poliquetos de Chile. Indice sinonímico y distribución geográfica de especies. Ediciones Universidad Católica de Chile, Ser. Mon. Biol., 3: 1- 284.

Rozbaczylo, N., T. Antezana \& H. Pinochet. 1987. Tomopteridae (Annelida: Polychaeta) recolectados en el Pacífico Suroriental y revisión de la familia en Chile. Stud. Neotrop. Fauna Environ., 22(2): 99 111. 
Støp-Bowitz, C. 1948. Polychaeta from the Michael Sars North Atlantic deep-sea expedition 1910. Rep. Scient. Results Michael Sars N. Atlant. Deep-Sea Exped., 5(8): 1-91.

Støp-Bowitz, C. 1949. Polychètes pélagiques des expéditions norvégiennes antarctiques de la Norvegia 1927-1928, 1928-1929 et 1930-1931. Det Norske Videnskaps-Akademi i Oslo. Scientific Results of the Norwegian Antarctic Expeditions 1927-1928 et sqq., Instituted and Financed by Consul Lars Christensen, 31: 3-25.

Støp-Bowitz, C. 1951. Polychètes pélagiques de l'Expédition Suédoise Antarctique 1901-1903. In Further Zoological Results of the Swedish Antarctic Expedition 1901-1903, 4(7): 1-14.

Tebble, N. 1960. The distribution of pelagic polychaetes in the South Atlantic Ocean. Discovery Rep., 30: 161-300.

Recibido: 12 marzo 2004; Aceptado: 19 julio 2004
Tebble, N. 1962. The distribution of pelagic polychaetes across the North Pacific Ocean. Bull. Brit. Mus.Nat. Hist. Zool., 7(9): 373-492.

Treadwell, A.L. 1906. Polychaetous annelids from the Hawaiian Islands, collected by the steamer Albatross in 1902. Bull. U.S. Fish. Com. Wash., 23: $1145-1181$.

Treadwell, A.L. 1928. Polychaetous annelids from the Arcturus Oceanographic Expedition. Zoologica, N.Y., 8: 449-489.

Treadwell, A.L. 1943. Biological results of the last cruise of the Carnegie. Polychaetous annelids. Scientific Results of Cruise VII of the Carnegie during 1928-1929 under the command of Captain J.P. Ault. Biology IV. Carn. Inst. Wash. Publ., 555: 31-59. 